EchoGéo

ECHOGEO-Sur le Vif | 2008

\title{
Les racines du dynamisme de la diaspora copte
}

\section{Grégoire Delhaye}

\section{OpenEdition}

\section{Journals}

Édition électronique

URL : http://journals.openedition.org/echogeo/6963

DOI : 10.4000/echogeo.6963

ISSN : 1963-1197

\section{Éditeur}

Pôle de recherche pour l'organisation et la diffusion de l'information géographique (CNRS UMR 8586)

\section{Référence électronique}

Grégoire Delhaye, «Les racines du dynamisme de la diaspora copte », EchoGéo [En ligne], Sur le Vif, mis en ligne le 22 août 2008, consulté le 03 mai 2019. URL : http://journals.openedition.org/ echogeo/6963 ; DOI : 10.4000/echogeo.6963

Ce document a été généré automatiquement le 3 mai 2019.

\section{(c) (i) (9)}

EchoGéo est mis à disposition selon les termes de la licence Creative Commons Attribution - Pas d'Utilisation Commerciale - Pas de Modification 4.0 International 


\title{
Les racines du dynamisme de la diaspora copte
}

\author{
Grégoire Delhaye
}

1 La baisse continue de la proportion de Coptes en Egypte est en partie attribuable à l'émigration permanente d'un nombre significatif d'entre eux. Celle-ci a pour corollaire le développement d'une diaspora particulièrement dynamique.

\section{Qui sont les Coptes?}

\subsection{Une église autocéphale issue d'un schisme ancien}

2 Evangélisée rapidement, l'Egypte était très majoritairement chrétienne à la fin du second siècle de notre ère. Après l'invasion arabe en 641, sa population se convertit progressivement à l'Islam et elle est aujourd'hui en grande majorité musulmane sunnite. Le pays abrite cependant une communauté de chrétiens autochtone de taille significative appartenant très majoritairement à l'église autocéphale copte issue d'un schisme ancien remontant au Concile de Chalcédoine en 451. Par commodité, en Egypte, le terme copte désigne généralement l'ensemble des chrétiens tous rites confondus. Bien que distincte de l'orthodoxie gréco-slave qu'elle précède de plus de cinq siècles, la chrétienté copte est souvent qualifiée d'orthodoxe pour la différencier des autres petites communautés chrétiennes égyptiennes notamment catholiques et protestantes issues des efforts missionnaires occidentaux à partir du XIX ${ }^{\mathrm{e}}$ siècle.

\subsection{La plus importante communauté chrétienne du Moyen-Orient}

3 Les chiffres des recensements égyptiens, bien que contestés depuis une quarantaine. d'années par les coptes, sont considérés comme fiables par les démographes (Courbage and Fargues, 1998, p.181). Ils montrent que bien que la population chrétienne égyptienne croisse très significativement en valeur absolue, elle décroît en proportion passant d'un million, ou $8 \%$ de la population, dans les années 1920 , à 3,34 millions, ou $5,7 \%$ de la 
population, en 1996, date des derniers chiffres rendus officiels. Ce même pourcentage appliqué aux estimations de la population égyptienne les plus récentes (CAPMAS, 2008), nous donne un chiffre de 4,2 millions de coptes. Cela fait des coptes, de loin, la plus importante communauté chrétienne au moyen-orient. Présents partout dans le pays ils ne sont, à l'exception de quelques rares villages, majoritaires nulle part. Pour un tiers cairotes et pour deux tiers provinciaux, leurs plus fortes concentrations sont en Moyenne-Egypte dans les gouvernorats de Minia, Assiout, Sohag et Qena. Ils sont en revanche très peu présents dans le Delta à l'exception d'Alexandrie (Denis, 2000).

\subsection{Un pape influent désormais loyal au régime}

4 Chenouda III, pape de l'Eglise Copte Orthodoxe depuis 1971, est très respecté par ses ouailles et exerce un contrôle serré sur l'institution. De fait, il est le seul à pouvoir légitimement représenter la communauté chrétienne dans l'espace public égyptien. Déchu et assigné à résidence en 1981 par Sadate pour avoir dénoncé l'islamisation du régime, il fut libéré en 1985 par Moubarak, l'actuel président. Il a depuis adopté une attitude conciliante vis-à-vis du régime allant jusqu'à soutenir publiquement l'autocrate égyptien lors des dernières élections présidentielles (Shahine, 2005). Cette loyauté lui permet d'obtenir certaines concessions des autorités. Il a ainsi récemment obtenu du gouvernement, au plus grand dam des autres rites chrétiens et de nombreux coptes orthodoxes, le durcissement de la législation en matière de divorce pour les chrétiens (AlKhatib, 2008).

\section{Discriminations plutôt que persécutions}

\subsection{Quelques discriminations d'ordre légal}

5 L'une des principales revendications des coptes a trait à l'entretien et à la construction des lieux de culte. En effet, jusqu'à récemment il fallait un décret présidentiel non seulement pour construire une nouvelle église ou un local paroissial mais également pour réparer un bâtiment existant. Bien qu'ayant été assouplie ces dernières années, l'obtention des permis est sujette aux bonnes relations du clergé local avec les autorités et avec la population locale ainsi qu'au feu vert des forces de sécurité qui souvent, de peur que des troubles n'éclatent, n'hésitent pas à mettre préventivement leur veto lorsque la majorité musulmane est hostile au projet. Une autre discrimination légale a trait à la reconnaissance légale des conversions: aisée vers l'islam elle est impossible de l'Islam vers toute autre religion (Guindy, 2008, p. 193). Cette disparité n'est pas sans conséquences pratiques. Certaines dispositions du droit, notamment en matière de mariage, de divorce et de droit de garde, s'appliquent en effet différemment selon la religion et sont généralement favorables aux musulmans (Guirguis, 2008, p.30-31).

\subsection{Des discriminations d'ordre social qui parfois dégénèrent}

6 Il existe surtout des discriminations sociales dont l'intensité et la fréquence varient grandement géographiquement. Sur le marché du travail et dans les administrations, les coptes sont parfois sujets à des abus de la part d'employeurs ou de fonctionnaires intolérants et il est, concrètement à l'heure actuelle, difficile d'obtenir justice ou 
réparation. Les coptes sont aussi parfois les victimes de violences qui peuvent aller de l'incendie au meurtre. Dans certains lieux où les tensions sont fortes, une simple dispute peut dégénérer en émeute sanglante comme en décembre 1999 à El-Kosheh (Marshall and Assad, 2001). Au-delà des stéréotypes sur «l'autre » dont se nourrit toute construction identitaire, les raisons de ces épisodes de violences sont complexes et peuvent avoir aussi bien trait à des usages politiciens de la variable confessionnelle comme ce fut le cas lors des émeutes d'Alexandrie en 2005, qu'à des dynamiques de classes se superposant aux tensions confessionnelles (Farah, 1986 ; Sedra, 1999) qu'attisent les inégalités rampantes dans l'Egypte contemporaine. Beaucoup moins nombreux, les coptes font les frais de ce climat tendu.

\subsection{Des autorités accusées d'inaction}

7 Face à l'intolérance dont sont victimes les coptes, le gouvernement reste relativement passif et répète à qui veut bien l'entendre que l'unité nationale est intacte. Afin de ne pas renforcer la contestation islamique, il joue l'apaisement et tente de prendre en compte les revendications coptes sans donner l'impression à la majorité musulmane de favoriser les chrétiens. Cela explique par exemple la clémence du verdict du procès des émeutes d'ElKosheh. Bien qu'elles firent 20 victimes chrétiennes pour une musulmane, seules deux personnes furent condamnées à des peines de prisons légères. Les services de sécurité furent également accusés d'avoir laissé les violences aller á leur terme avant d'intervenir. C'est ce genre de manquement qui font dire à ses détracteurs que c'est l'état qui est responsable de tous les maux des Coptes. La question copte est un sujet tellement sensible qu'elle est l'une des seules à avoir longtemps été activement censurée par le pouvoir (Middle East Times, 2005). Ces dernières années cependant, le débat s'est libéré en raison notamment d'une actualité confessionnelle chargée (El-Amrani, 2006). Désormais les médias et notamment la presse écrite gouvernementale admettent l'existence de problèmes d'ordre confessionnel : ils les dénoncent et insistent sur la nécessité d'une gestion locale de chacun d'entre eux.

\subsection{Des gestes positifs des autorités}

Les autorités égyptiennes ont également pris récemment plusieurs mesures positives en faveur de la communauté copte. La législation sur les lieux de cultes a été amendée à plusieurs reprises afin de mettre à terme, toutes les religions sur un pied d'égalité (Guirguis, 2008, p. 47, note 29) et en 2002, le Noël copte, célébré le 7 janvier, est devenu une fête nationale chômée par tous. Dans un contexte égyptien marqué, malgré des réformes de façade, par l'autoritarisme (Kienle, 2001), la légitimité du régime est limitée et tout geste vis-à-vis des chrétiens peut rapidement être dénoncé par la fraction la plus intolérante de l'opposition islamique comme un signe d'indifférence du régime vis-à-vis de la majorité musulmane. Ces mesures administratives ne peuvent être dès lors comprises que dans le contexte du déclin, amorcé en 1977, des groupes islamistes les plus radicaux et de l'amélioration significative de la situation sécuritaire qui en découle mais également des pressions internationales, en particulier américaines, soutenues par une frange militante de la diaspora copte minoritaire mais d'autant plus bruyante que la distance la protège de l'ire de l'état policier. Le pape Chenouda se contente de son coté de dénoncer mollement les militants dont l'intransigeance renforcent sa stature 
d'interlocuteur modéré (c'est le radical flank effect abordé notamment par McAdam et al., 1996, p. 14).

\section{L'émergence d'une diaspora copte dynamique}

\subsection{Une émigration principalement économique}

9 Le caractère essentiellement économique de l'émigration égyptienne a été établi par plusieurs auteurs (Talani et al., 2003 ; Zohry, 2003 ; Talani, 2005). Plusieurs études qualitatives et mes travaux de doctorat suggèrent qu'il en est de même en ce qui concerne plus particulièrement l'émigration copte (Abdelnour, 1993, p. 3 ; Botros, 2005, p. 54). Rares sont en effet les coptes interviewés sur les causes de leur départ, que ce soit vers la France ou les Etats-Unis qui n'aient mentionné en premier le manque d'opportunités professionnelles. Généralement entendu comme touchant l'ensemble d'une société égyptienne ou règne la loi de "wasta », ou piston (Cunningham and Sarayrah, 1994), la manque d'opportunité fut éventuellement lié en partie, dans un second temps, à des discriminations d'ordre confessionnel. Botros dans son enquête tire globalement les mêmes conclusions en insistant plus particulièrement sur le rôle des discriminations religieuses dans la détérioration de la situation socioéconomique des coptes en Egypte (2005, p. 47 et suiv.).

\subsection{Des pays d'immigration différents de ceux des musulmans égyptiens}

10 Si les causes de l'émigration sont donc globalement similaires pour coptes et musulmans égyptiens, les pays d'immigration varient significativement en fonction de la religion du migrant. Les pays du Golfe, en particulier l'Arabie Saoudite et, jusqu'en 1991, l'Irak, sont en effet la destination privilégiée des égyptiens (Nassar, 2007, p. 60). Cependant les différents annuaires web recensant les églises coptes orthodoxes sont sur ce point riches d'enseignements. Leur distribution géographique laisse ainsi clairement apparaître un tropisme occidental et en particulier nord-américain des migrations coptes. Il n'existe ainsi aujourd'hui pas moins de 25 paroisses coptes au Canada, 130 aux Etats-Unis dont 32 pour la seule Californie (Coptic Othodox Church Network, 2008), plusieurs dizaines en Europe et en Australie. La diaspora a également fondé plusieurs monastères et séminaires coptes en dehors de l'Egypte, preuve supplémentaire d'un dynamisme émanant de la capacité de l'institution ecclésiale à maintenir à l'étranger ses ouailles impliquées dans son fonctionnement.

\subsection{Une socialisation immigrante centrée sur les églises}

11 S'il n'existe donc pas vraiment d'émigration spécifiquement copte, les facteurs poussant les coptes égyptiens à partir étant les mêmes que ceux de leurs co-nationaux musulmans, il existe en revanche clairement une immigration copte (sur la distinction voir Sayad, 1999, p. 15 et suiv.) . Les paroisses coptes jouent en effet un rôle central dans l'expérience migratoire copte aussi bien aux Etats-Unis (Abdelnour, 1993) et au Canada (Botros, 2005) qu'en France (Ayad, 1989). Elles fournissent une assistance concrète aux nouveaux venus, qui va de l'aide aux formalités à l'accès à des logements temporaires (Abouna Anthony, 
2003) mais offrent également espace de socialisation qui n'est pas sans rappeler, dans son perpétuel ballet d'activités, les paroisses coptes en Egypte (voir par exemple Vivier, 2000). La paroisse copte de Fairfax, à côté de Washington accueille ainsi, en plus de l'église ellemême, une école primaire en journée, des cours d'arabe, des groupes de prière ou de catéchèse pour tous les âges, une salle de sport, une salle de réception et une immense cuisine. La paroisse de Villejuif près de Paris, est également le théâtre de nombreuses activités, notamment d'une bourse à l'emploi informelle.

\subsection{Les racines du dynamisme de la diaspora copte}

12 L'histoire moderne de l'église copte a été marquée, je l'ai évoqué rapidement, par un important renouveau identitaire et institutionnel. Celui-ci ne fut pas tant le produit de la montée de la violence islamiste des année 1970-1980 que le fruit d'un long processus de réforme plus ancien initié au XIX ${ }^{\mathrm{e}}$ siècle (El-Khawaga, 1992 ; Sedra, 2004 ; Van DoornHarder, 2005) et déclenché, tout comme le renouveau islamique, par la présence coloniale en Egypte. Le développement, caractéristique du renouveau, des prestations offertes par les paroisses s'est combiné à l'augmentation de la demande pour ses services sous les des effets combinés de la montée en puissance des discriminations confessionnelles et de l'échec de l'état providence qui a contribué à l'isolation croissante des coptes en Egypte. Ces même pratiques communautarisantes, qui participent de l'aliénation croissante des coptes en Egypte, reproduites et adaptées dans le contexte diasporique se révèlent être particulièrement propices à la préservation du lien communautaire. Les paroisses bénéficient dans leur tache du soutien d'une institution ecclésiale dont la centralisation et l'efficacité sont, elles aussi, des produits du renouveau

\section{BIBLIOGRAPHIE}

Abdelnour Ziad, 1993. Le rôle politique de la diaspora copte d'amérique Du Nord. In Section: Analyse du monde arabe contemporain, p. 65 + annexes. Paris: Institut d'Etude Politiques.

Abouna Anthony 2003. Entretien Individuel (Prêtre de la paroisse copte de Fairfax).

Al-Khatib Ahmed, 2008. Al-Masry Al-Youm Unveils the Chrurch's New Act of Personal Affairs to Be Issued with the Government's Consent. Al-Masry Al-Youm Online (May 21) Translated from arabic by Eltorjoman International

(http://almasry-alyoum.com/article2.aspx?articleID=106115)

Ayad Christophe, 1989. La Communauté Copte à Paris: Compte rendu d'enquête. Séminaire de Science Politique Niveau III, p. 59. Paris: Institut d'Etudes Politiques.

Botros Ghada Barsoum, 2005. Competing for the Future: Adaptation and the Accommodation of Difference in Coptic Immigrant Churches. p. 225: University of Toronto (Canada).

CAPMAS, 2008. Estimates of Population. Central Agency for Mobilization and Statistics of Egypt ( http://www.capmas.gov.eg/eng_ver/sdds/POPULATION.htm) Accessed: 2008 May, 29 
Coptic Othodox Church Network, 2008. Directory of Churches in the U.S. And Canada. www.copticchurch.net Accessed: 2008 June

Courbage Youssef and Philippe Fargues, 1998. Christians and Jews under Islam. London, I.B. Tauris. Cunningham Robert B. and Yasin K Sarayrah, 1994. Taming Wasta to Achieve Development. Arab Studies Quarterly 16(3), p. 29.

Denis Eric, 2000. Cent ans de localisation de la population chrétienne égyptienne. Astrolabe (2).

El-Amrani Issandr, 2006. The Emergence of a "Coptic Question" in Egypt. MERIP/Middle East Report Online (http://www.merip.org/mero/mero042806.html)

El-Khawaga Dina, 1992. Le développement communautaire copte: Un mode de participation au politique. Magreb-Machrek (135).

Farah Nadia Ramsis, 1986. Religious Strife in Egypt: Crisis and Ideological Conflict in the Seventies. New York: Gordon and Breach Science Publishers.

Guindy Adel, 2008. Le droit de croyance en Égypte: entre liberté et coercition. In Conversions religieuses et mutations Politiques, edited by Laure Guirguis, p. 175-198. Paris: Non Lieu.

Guirguis Laure, 2008. La conversion celigieuse cn Égypte: Lieu des impasses et des Possibles. In Conversions religieuses et mutations politiques, edited by Laure Guirguis, p. 21-50. Paris.

Kienle Eberhard, 2001. A Grand Delusion: Democracy and Economic Reform in Egypt. London; New York: I.B. Tauris.

Marshall Paul and Joseph Assad, 2001. Massacre at the Millennium. p. 131. Washington D.C., Center for Religious Freedom, Freedom House.

McAdam Doug, John D. McCarthy and Mayer N. Zald, 1996. Comparative Perspectives on Social Movements: Political Opportunities, Mobilizing Structures, and Cultural Framings. Cambridge Studies in Comparative Politics. Cambridge England; New York: Cambridge University Press.

Middle East Times. 2005. Censored Articles in Egypt. (http://www.metimes.com/censored/)

Nassar Heba, 2007. Egypt: The Demographic and Economic Dimension of Migration. In Migrations Méditerranéennes: Rapport 2006-2007, edited by Philippe Fargues, p. 411. Florence, CARIM.

Sayad Abdelmalek, 1999. La double absence. Paris, Seuil.

Sedra Paul, 1999. Class Cleavages and Ethnic Conflict: Coptic Christian Communities in Modern Egyptian Politics. Islam \& Christian-Muslim Relations 10(2): p 219-36.

Sedra Paul, 2004 Review of "Christians Versus Muslims in Modern Egypt: The Century-Long Struggle for Coptic Equality" by Sana Hasan. Middle East Journal 58(3) p. 510-511.

Shahine Gihan, 2005. Jumbled Reactions. Al-Ahram Weekly (756).

Talani Leila S, 2005 Out of Egypt: Globalisation, Marginalisation and Illegal Muslim Migration to the Eu. In Occasional Lecture Series, p. 43. Los Angeles: UCLA Center for European and Eurasian Studies.

Talani Leila Simona, Stefan Wolff, Kirstin Henrard and Eiko Thielemann, 2003.Why Do Migrants Leave Their Countries? Motivations to Migrate at the Point of Departure: The Case of Egypt. p. 71. Cairo: Egyptian Ministry for Manpower and Emigration.

Van Doorn-Harder, Pieternella, 2005, Copts: Fully Egyptian, but for a Tattoo? In Nationalism and Minority Identities in Islamic Societies, edited by Maya Shatzmiller, p. 22-57. Montreal; Ithaca: McGill-Queen's University Press. 
Vivier Anne-Sophie, 2000. Coptes orthodoxes d'egypte: discours et pratiques identitaires. In Département de Sociologie, p. 159. Paris: Université Paris X.

Zohry Ayman Gaafar, 2003. Contemporary Egyptian Migration 2003.p. 98, International Organization for Migration.

\section{RÉSUMÉS}

Les coptes, la minorité chrétienne autochtone d'Egypte, doivent faire face à des discriminations principalement d'ordre social mais également dans une certaine mesure d'ordre légal dans leur pays. Ayant connu un renouveau institutionnel et religieux important depuis le XIX ${ }^{\mathrm{e}}$ siècle, les mécanismes de préservation communautaire qui en sont issus en Egypte, une fois transposés dans le contexte diasporique se révèlent particulièrement efficaces pour résister à l'acculturation.

Copts, Egypt indigenous Christian minority, face some legal but mostly social discrimination in their homeland. Having experienced a significant institutional and spiritual revival since the $19^{\text {th }}$ century, the ensuing preservation mechanism developed in Egypt, once transposed, in the diasporic context turn out particularly efficient in resisting acculturation.

\section{INDEX}

Mots-clés : Coptes, Egypte, minorité, discrimination, migration, diaspora

Keywords : Copts, Egypt, minority, discrimination, migration, diaspora

\section{AUTEUR}

\section{GRÉGOIRE DELHAYE}

Grégoire Delhaye (gregoire.delhaye@gmail.com) est doctorant en science politique )à l'IEP d'Aix en Povence/IREMAM et enseigne la science politique comparative à American University à Washington DC. Ses travaux explorent les enjeux et les contraintes des mobilisations politiques diasporiques à travers le cas du militantisme coptes aux Etats-Unis.Il a récemment publié « La réponse des états à la dissidence diasporique : Le cas de l'Egypte face au militantisme copte aux Etats-Unis » dans Stéphane Dufoix, dir., Loin des yeux, près du cœur ? Formes et évolutions des politiques étatiques envers les populations nationales vivant en dehors des frontières, Presses de Sciences-Po, Septembre 2008 ainsi que « Comprendre la mondialisation des normes : Les leçons de l'échec de la liberté religieuse universelle » dans Yves Schemeil et Wolf-Dieter Eberwein, dir. Normer le monde : création et réception des normes internationales, L'Harmattan, Octobre 2008. 